\title{
ON THE COMPARISON OF POSITIVE ELEMENTS OF A C*-ALGEBRA BY LOWER SEMICONTINUOUS TRACES
}

\author{
LEONEL ROBERT
}

\begin{abstract}
It is shown in this paper that two positive elements of a $\mathrm{C}^{*}$ algebra agree on all lower semicontinuous traces if and only if they are equivalent in the sense of Cuntz and Pedersen. A similar result is also obtained in the more general case where the two elements are comparable by their values on the lower semicontinuous traces. This result is used to give a characterization of the functions on the cone of lower semicontinuous traces of a stable $\mathrm{C}^{*}$-algebra that arise from positive elements of the algebra.
\end{abstract}

\section{INTRODUCTION}

In 1], Cuntz and Pedersen considered the problem of comparing positive elements of a $\mathrm{C}^{*}$-algebra by their values on the lower semicontinuous traces on the algebra. They defined an equivalence relation on the positive elementsthe Cuntz-Pedersen relation - and showed, among other results, that if the $\mathrm{C}^{*}$ algebra is simple, then two positive elements are Cuntz-Pedersen equivalent if and only if they agree on all the lower semicontinuous traces. The question whether this was true for an arbitrary $\mathrm{C}^{*}$-algebra was left unsettled in their paper, and is answered affirmatively in Theorem 1 below.

Let $A$ be $\mathrm{C}^{*}$-algebra. Recall that a trace on $A$ is a map $\tau: A^{+} \rightarrow[0, \infty]$ that is additive, homogeneous, and satisfies the identity $\tau\left(x x^{*}\right)=\tau\left(x^{*} x\right)$. We will be mostly interested in the lower semicontinuous traces on $A$, the set of which we shall denote by $T(A)$.

For $a, b \in A^{+}$let us say that $a$ is Cuntz-Pedersen equivalent to $b$, denoted by $a \sim b$, if $a=\sum_{i=1}^{\infty} x_{i} x_{i}^{*}$ and $b=\sum_{i=1}^{\infty} x_{i}^{*} x_{i}$ for some $x_{i} \in A$. Let us say that $a$ is Cuntz-Pedersen smaller than $b$, and denote this by $a \preceq b$, if $a \sim a^{\prime} \leq b$ for some $a^{\prime} \in A^{+}$. It was shown in [1] that the relations $\sim$ and $\preceq$ are transitive (so $\sim$ is an equivalence relation).

Theorem 1. Let $a$ and $b$ be positive elements of $A$. The following propositions are true.

(i) $\tau(a) \leq \tau(b)$ for all $\tau \in T(A)$ if and only if for every $\epsilon>0$ there is $\delta>0$ such that $(a-\epsilon)_{+} \preceq(b-\delta)_{+}$.

(ii) $\tau(a)=\tau(b)$ for all $\tau \in T(A)$ if and only if $a \sim b$.

Remark. We shall see in Section 3 an example where $\tau(a) \leq \tau(b)$ for all $\tau \in T(A)$, but it is not true that $a \preceq b$.

2000 Mathematics Subject Classification. 46L05, 46L35. 


\section{Proof of Theorem 1}

The proof of Theorem 1 is preceded by a number of preliminary definitions and results.

Let $a, b \in A^{+}$. Let us write $a \preceq_{C P} b$ if for every $\epsilon>0$ there is $\delta>0$ such that $(a-\epsilon)_{+} \preceq(b-\delta)_{+}$. Since $\preceq$ is transitive, the relation $\preceq_{C P}$ is clearly transitive as well. Let us write $a \sim_{C P} b$ if $a \preceq_{C P} b$ and $b \preceq_{C P} a$. This defines an equivalence relation in $A^{+}$. (It will be shown in the proof of Theorem 1 (ii) that $\sim_{C P}$ is the same as $\sim$.)

Proposition 1. Let $a, b, c, d \in A^{+}$. The following propositions are true.

(i) If $a \preceq_{C P} b$ and $c \preceq_{C P} d$ then $a+c \preceq_{C P} b+d$.

(ii) If $a \preceq_{C P} b$ then $\alpha a \preceq_{C P} \alpha b$ for all $\alpha \in \mathbb{R}^{+}$.

(iii) If $a \preceq b$ then $a \preceq_{C P} b$.

Proof. It was shown in [2, Proposition 2.3] that for all $a, b \in A^{+}$and $\epsilon>0$ there is $\delta>0$ such that

$$
\begin{aligned}
(a-\epsilon)_{+}+(b-\epsilon)_{+} & \preceq(a+b-\delta)_{+}, \\
(a+b-\epsilon)_{+} & \preceq(a-\delta)_{+}+(b-\delta)_{+} .
\end{aligned}
$$

These inequalities imply (i).

(ii) This is clear.

(iii) Suppose that $a=\sum_{i=1}^{\infty} x_{i} x_{i}^{*}$ and $\sum_{i=1}^{\infty} x_{i}^{*} x_{i} \leq b$. Let $\epsilon>0$. By the lemma of Kirchberg and Rørdam [3, Lemma 2.2] (see also the remark after [2, Lemma 2.2]), there are $n \in \mathbb{N}$ and $\epsilon_{1}>0$ such that $(a-\epsilon)_{+} \preceq$ $\left(\sum_{i=1}^{n} x_{i} x_{i}^{*}-\epsilon_{1}\right)_{+}$. We have

$$
\begin{aligned}
(a-\epsilon)_{+} \preceq\left(\sum_{i=1}^{n} x_{i} x_{i}^{*}-\epsilon_{1}\right)_{+} \preceq \sum_{i=1}^{n}\left(x_{i} x_{i}^{*}-\epsilon_{2}\right)_{+} & \sim \sum_{i=1}^{n}\left(x_{i}^{*} x_{i}-\epsilon_{2}\right)_{+} \\
& \preceq\left(\sum_{i=1}^{n} x_{i} x_{i}^{*}-\epsilon_{3}\right)_{+} \preceq\left(b-\epsilon_{4}\right)_{+} .
\end{aligned}
$$

In the above chain of inequalities we have applied (1), (2) and that $\left(x x^{*}-\epsilon\right)_{+} \sim$ $\left(x^{*} x-\epsilon\right)_{+}$for all $\epsilon>0$ (see [2, Proposition 2.3]).

Let us denote by $A_{C P}$ the quotient $A^{+} / \sim_{C P}$. We consider $A_{C P}$ ordered by the order $\langle a\rangle \leq\langle b\rangle$ if $a \preceq_{C P} b$, where $\langle a\rangle$ and $\langle b\rangle$ denote the equivalence classes of the positive elements $a$ and $b$. We also endow $A_{C P}$ with the addition operation $\langle a\rangle+\langle b\rangle:=\langle a+b\rangle$. The order of $A_{C P}$ is compatible with the addition operation, i.e., $\langle a\rangle \leq\langle a\rangle+\langle b\rangle$. Thus, $A_{C P}$ is an ordered semigroup with 0 .

In order to prove Theorem 1 (i) we will apply the following proposition to the ordered semigroup $A_{C P}$.

Proposition 2. ([4, Proposition 3.2]) Let $S$ be an ordered semigroup with 0 and with the property that if $(k+1) x \leq k y$ for some $x, y \in S$ and $k \in \mathbb{N}$, then $x \leq y$ (i.e., $S$ is almost unperforated). The following implication holds in $S$ :

If $x \leq M y$ for some $M \in \mathbb{N}$, and $\lambda(x)<\lambda(y)$ for every $\lambda: S \rightarrow[0, \infty]$ that is additive, order-preserving, and satisfies $\lambda(y)=1$, then $x \leq y$. 
Notice that the semigroup $A_{C P}$ satisfies the hypotheses of the preceding proposition. In fact, in $A_{C P}$ we have that $k\langle x\rangle \leq k\langle y\rangle$ implies $\langle x\rangle \leq\langle y\rangle$, because we can multiply the elements of $A_{C P}$ by positive real scalars.

Notice also that for every additive map $\lambda: A_{C P} \rightarrow[0, \infty]$, the map on $A^{+}$ defined by $\tau_{\lambda}(a):=\lambda(\langle a\rangle)$ is a trace, because it is additive and satisfies the trace identity (homogeneity holds automatically for any additive map with values in $[0, \infty])$. This trace may not be lower semicontinuous. To Theorem [1 (i) we will then need the following lemma.

Lemma 1. (2, Lemma 3.1]) Let $\tau: A^{+} \rightarrow[0, \infty]$ be a trace on the $C^{*}$-algebra A. Then $\tilde{\tau}(a)=\sup _{\epsilon>0} \tau\left((a-\epsilon)_{+}\right)$is a lower semicontinuous trace.

Proof of Theorem 1 ( $i)$. It is clear that if $a \preceq_{C P} b$ then $\tau(a) \leq \tau(b)$ for every $\tau \in T(A)$.

Let us assume that $\tau(a) \leq \tau(b)$ for all $\tau \in T(A)$. For every closed two-sided ideal $I$ of $A$, the map defined by $\tau_{I}(x)=0$ if $x \in I^{+}$and $\tau_{I}(x)=\infty$ otherwise, is a lower semicontinuous trace on $A$. Since $\tau_{I}(a) \leq \tau_{I}(b)$ for any such trace it follows that $\operatorname{Ideal}(a) \subseteq \operatorname{Ideal}(b)$. Let $\epsilon>0$. We have $(a-\epsilon)_{+} \in \operatorname{Ped}(\operatorname{Ideal}(b))^{+}$; hence $(a-\epsilon)_{+}=\sum_{i=1}^{m} y_{i} b y_{i}^{*}$ for some $y_{i} \in A$. This implies that $(a-\epsilon)_{+} \preceq M b$ for some $M \in \mathbb{N}$, and so $\left\langle(a-\epsilon)_{+}\right\rangle \leq M\langle b\rangle$. Let us show that we also have $\lambda\left(\left\langle(a-\epsilon)_{+}\right\rangle\right)<\lambda(\langle b\rangle)$ for any additive, order-preserving map $\lambda: A_{C P} \rightarrow[0, \infty]$ such that $\lambda(\langle b\rangle)=1$. By Proposition 2, this will imply that $\left\langle(a-\epsilon)_{+}\right\rangle \leq\langle b\rangle$, and since $\epsilon$ is arbitrary, we will have $\langle a\rangle \leq\langle b\rangle$, as desired.

Let $\lambda: A_{C P} \rightarrow[0, \infty]$ be additive, order-preserving, and such that $\lambda(\langle b\rangle)=$ 1. Let $\tau_{\lambda}$ be the trace associated to $\lambda$ and $\tilde{\tau}_{\lambda}$ its lower semicontinuous regularization defined as in Lemma 1. Notice that for every $\delta>0$ and $c \in A^{+}$we have $\tau_{\lambda}\left((c-\delta)_{+}\right) \leq \tilde{\tau}_{\lambda}(c) \leq \tau_{\lambda}(c)$.

Case 1. Assume that $\tilde{\tau}_{\lambda}(a) \neq 0$. Then

$\lambda\left(\left\langle(a-\epsilon)_{+}\right\rangle\right)=\tau_{\lambda}\left((a-\epsilon)_{+}\right) \leq \tilde{\tau}_{\lambda}\left((a-\epsilon / 2)_{+}\right)<\tilde{\tau}_{\lambda}(a) \leq \tilde{\tau}_{\lambda}(b) \leq \tau_{\lambda}(b)=\lambda(\langle b\rangle)$.

Case 2. Assume that $\tilde{\tau}_{\lambda}(a)=0$. Then

$$
\lambda\left(\left\langle(a-\epsilon)_{+}\right\rangle\right)=\tau_{\lambda}\left((a-\epsilon)_{+}\right)=0<1=\lambda(\langle b\rangle) .
$$

We now turn to the proof of Theorem 1 (ii).

Lemma 2. Let $a, b, c \in A^{+}$. The following propositions are true.

(i) If $a+c \preceq b+c$ and $c \preceq M b$ for some $M \in \mathbb{R}^{+}$, then $a \preceq(1+\delta) b$ for all $\delta>0$.

(ii) If $a+c \sim b+c$ and $c \preceq M a, M b$ for some $M \in \mathbb{R}^{+}$, then $a \sim b$.

Proof. (i) Adding $a$ on both sides of $a+c \preceq b+c$ we obtain that $2 a+c \preceq 2 b+c$, and by induction $k a+c \preceq k b+c$ for all $k$. Dividing the last inequality by $k$ we see that we may assume that $c \preceq b$. Changing $c$ to $c^{\prime}$ such that $c \sim c^{\prime} \leq b$ we may further assume that $c \leq b$. We have $a+b=a+c+(b-c) \preceq 2 b$. By applying induction we obtain that $k a+b \preceq(k+1) b$ for all $k$. This proves (i).

(ii) As in the proof of (i) we may assume that $c \leq b$. We have $a+b \sim$ $a+c+b-c \sim 2 b$. In the same way we obtain that $a+b \sim 2 a$. Therefore, $a \sim b$. 
Proof of Theorem 1 (ii). By Theorem 1 (i) we know that $a \preceq_{C P} b$ and $b \preceq_{C P} a$. Thus, there are strictly decreasing sequences of positive numbers $\epsilon_{n}$ and $\mu_{n}$ such that $\left(a-\epsilon_{n}\right)_{+} \preceq\left(b-\mu_{n}\right)_{+} \preceq\left(a-\epsilon_{n+1}\right)_{+}$for all $n \in \mathbb{N}$, and $\epsilon_{n}, \mu_{n} \rightarrow 0$. Let us write $a_{n}=\left(a-\epsilon_{n}\right)_{+}$and $b_{n}=\left(b-\mu_{n}\right)$. The proof now follows the same arguments as in [1, Lemma 7.4], although with some modifications.

Since $a_{n} \preceq b_{n}$ there is $a_{n}^{\prime}$ such that $a_{n} \sim a_{n}^{\prime} \leq b_{n}$. We have $\left(b_{n}-a_{n}^{\prime}\right)+a_{n}^{\prime} \preceq$ $\left(a_{n+1}-a_{n}\right)+a_{n}$. We also have $a_{n} \leq M\left(a_{n+1}-a_{n}\right)$. (This is verified by referring back to the definition of $a_{n}$ in terms of $a$.) By Lemma 2 (i) we have that $b_{n}-a_{n}^{\prime} \preceq(1+\delta)\left(a_{n+1}-a_{n}\right)$ for all $\delta>0$. For $\delta$ small enough we have that $b_{n}-a_{n}^{\prime} \preceq(1+\delta)\left(a_{n+1}-a_{n}\right) \leq a_{n+2}-a_{n}$. Let $z$ be such that $b_{n}-a_{n}^{\prime} \sim z \leq a_{n+2}-a_{n}$ and let $b_{n}^{\prime}=a_{n}+z$. The sequence $\left(b_{n}^{\prime}\right)$ satisfies that $b_{n}^{\prime} \sim b_{n}$ and $a_{n} \leq b_{n}^{\prime} \leq a_{n+2}$.

We have $\left(b_{n+3}^{\prime}-b_{n}^{\prime}\right)+b_{n}^{\prime} \sim\left(b_{n+3}-b_{n}\right)+b_{n}$. Let us see that we can apply Lemma 2 (ii) to conclude that $\left(b_{n+3}^{\prime}-b_{n}^{\prime}\right) \sim\left(b_{n+3}-b_{n}\right)$. On one hand, $b_{n} \leq M\left(b_{n+3}-b_{n}\right)$ for some $M>0$, by the expression of these elements in terms of the functional calculus of $b$. On the other hand, $b_{n}^{\prime} \leq a_{n+2} \leq$ $M^{\prime}\left(a_{n+3}-a_{n+2}\right) \leq M^{\prime}\left(b_{n+3}^{\prime}-b_{n}^{\prime}\right)$ for some $M^{\prime}>0$. Hence, Lemma 2 (ii) can be applied. We have

$$
a=b_{0}^{\prime}+\sum_{k=1}^{\infty}\left(b_{3(k+1)}^{\prime}-b_{3 k}^{\prime}\right) \sim b_{0}+\sum_{k=1}^{\infty}\left(b_{3(k+1)}-b_{3 k}\right)=b .
$$

\section{FURTHER REMARKS}

Let us first show that Theorem 1 (i) cannot be strengthened to obtain $\tau(a) \leq \tau(b)$ for all $\tau$ implies $a \preceq b$.

Example. Let $A$ be a simple unital $\mathrm{C}^{*}$-algebra with exactly two extreme tracial states $\tau_{1}$ and $\tau_{2}$. Such an algebra may be found, say, among the AF $\mathrm{C}^{*}$-algebras (e.g., [1, Example 6.10]). Let $b \in A^{+}$be such that $\tau_{1}(b)<\tau_{2}(b)$ and set $\tau_{1}(b) 1=a$. Then $\tau_{1}(a)=\tau_{1}(b)$ and $\tau_{2}(a)<\tau_{2}(b)$, and so for every bounded trace $\tau, \tau(a) \leq \tau(b)$. This also holds for the unique unbounded trace with value $\infty$ on all points except 0 . But we cannot have $a \preceq b$, because in that case $a+c \sim b$ for some $c \in A^{+}$, and so $\tau_{1}(c)=0$ and $\tau_{2}(c)>0$, which is impossible by the simplicity of $A$.

Let us consider the following topology on $T(A)$ : the net of lower semicontinuous traces $\left(\tau_{i}\right)$ converges to $\tau$ if

$$
\lim \sup \tau_{i}\left((a-\epsilon)_{+}\right) \leq \tau(a) \leq \liminf \tau_{i}(a)
$$

for all $a \in A^{+}$and $\epsilon>0$. It was shown in [2] that $T(A)$ is a compact Hausdorff space and that the functor $T(\cdot)$ is a continuous contravariant functor from the category of $\mathrm{C}^{*}$-algebras to the category of topological spaces.

For every positive element $a \in A^{+}$the function $\widetilde{a}: T(A) \rightarrow[0, \infty]$, defined by $\widetilde{a}(\tau)=\tau(a)$, is linear and lower semicontinuous. Consider the following question: Which linear and lower semicontinuous functions on $T(A)$ arise from positive elements of $A$ in the form $\widetilde{a}$ ? In view of Theorem 1, this question is asking for a description of the ordered semigroup $A_{C P}$ as a semigroup of 
functions on $T(A)$. Theorem 2 below gives an answer to this question assuming that $A$ is stable.

Let us recall the definition of the non-cancellative cone $S(T(A))$ given in [2]. Let $\operatorname{LSC}(T(A))$ denote the linear, lower semicontinuous, functions on $T(A)$ with values in $[0, \infty]$. Then $S(T(A))$ is composed of the functions $f \in L S C(T(A))$ that satisfy the following condition: there is an increasing sequence $\left(h_{n}\right), h_{n} \in L S C(T(A))$, such that $f=\sup h_{n}$ and $h_{n}$ is continuous on each point where $h_{n+1}$ is finite.

For every $a \in A^{+}$we have $\widetilde{a} \in S(T(A)$ ) (see [2, Proposition 5.1]). Moreover, by [2, Theorem 5.9], $S(T(A))$ is the set of functions $f \in L S C(T(A))$ for which there is an increasing sequence of functions $\widetilde{a}_{n}$ coming from positive elements $a_{n} \in A^{+}$and such that $f=\sup \widetilde{a}_{n}$. We can now prove the following theorem.

Theorem 2. Let $A$ be a stable $C^{*}$-algebra. Then $S(T(A))$ is the set of functions of the form $\widetilde{a}$ for some $a \in A^{+}$.

Proof. Let $f$ be in $S(T(A))$ and let $\left(\widetilde{a_{n}}\right)$, with $a_{n} \in A^{+}$, be an increasing sequence of functions with supremum $f$. By Theorem 1 (i), we have that $a_{n} \preceq_{C P} a_{n+1}$ for all $n$. We may replace the positive elements $a_{n}$ by $a_{n}^{\prime}=$ $\left(a_{n}-\epsilon_{n}\right)_{+}$, with $\epsilon_{n}>0$ small enough, so that $a_{n}^{\prime} \preceq a_{n+1}^{\prime}$ and $f=\sup \widetilde{a_{n}^{\prime}}$. Let $c_{n} \in A^{+}$be such that $a_{n}^{\prime}+c_{n} \sim a_{n+1}^{\prime}$. Using the stability of $A$ we may find mutually orthogonal elements $c_{n}^{\prime \prime}$ such that $c_{n}^{\prime \prime} \sim c_{n}^{\prime}$. Furthermore, again by the stability of $A$, we may assume that the $c_{n}^{\prime \prime}$ s have sufficiently small norm such that the series $a=\sum_{n=1}^{\infty} c_{n}^{\prime \prime}$ converges. We then have $f=\widetilde{a}$.

Question 1. Is $S(T(A))=L S C(T(A))$ ?

Question 2. In [2] a cone dual to the 2-quasitraces of $A$ is defined which is analogous to the cone $S(T(A))$. Is the analog of Theorem 2 for 2-quasitraces true?

Acknowledgments. I am grateful to George A. Elliott for pointing out that Theorem 2 was in all likelihood true.

\section{REFERENCES}

[1] J. Cuntz and G. K. Pedersen, Equivalence and traces on $C^{*}$-algebras, J. Funct. Anal. 33 (1979), no. 2, 135-164.

[2] G. A. Elliott, L. Robert, and L. Santiago, On the cone of lower semicontinuous traces on a $C^{*}$-algebra, arXiv:0805.3122v1 [math. OA] (2008).

[3] E. Kirchberg and M. Rørdam, Infinite non-simple $C^{*}$-algebras: absorbing the Cuntz algebras $\mathcal{O}_{\infty}$, Adv. Math. 167 (2002), no. 2, 195-264.

[4] M. Rørdam, The stable and the real rank of $\mathcal{Z}$-absorbing $C^{*}$-algebras, Internat. J. Math. 15 (2004), no. 10, 1065-1084.

Leonel Robert, Fields Institute, Toronto, Canada, M5T 3J1

E-mail address: lrobert@fields.utoronto.ca 